\title{
Indirect Analogical Reasoning Components
}

\author{
Kristayulita* \\ Faculty of Tarbiyah and Teachers Training, Universitas Islam Negeri Mataram, Mataram, Indonesia, 83125 \\ *Corresponding author: kristayulita@uinmataram.ac.id | Phone Number: +6285228131785
}

\section{ARTICLE HISTORY}

Received : 28 September 2020

Revised : 11 March 2021

Accepted : 10 April 2021

\section{KEYWORDS}

Analogical reasoning; Component;

Indirect analogical problems;

\begin{abstract}
If using different instruments obtained a different analogical reasoning component. With use peoplepiece analogies, verbal analogies, and geometric analogies, have analogical reasoning component consists of encoding, inferring, mapping, and application. Meanwhile, with use analogical problems (algebra, source problem and target problem is equal), have analogical reasoning components consist of structuring, mapping, applying, and verifying. The instrument used was analogical problems consisting of two problems where the source problem was symbolic quadratic equation problem and the target problems were trigonometric equation problem and a word problem. This study aims to provide information analogical reasoning process in solving indirect analogical problems. in addition, to identify the analogical reasoning components in solving indirect analogical problems. Using a qualitative design approach, the study was conducted at two schools in Mataram city of Nusa Tenggara Barat, Indonesia. The results of the study provide an overview of analogical reasoning of the students in solving indirect analogical problems and there is a component the representation and mathematical model in solving indirect analogical problems. So the analogical reasoning component in solving indirect analogical problems is the representation and mathematical modeling, structuring, mapping, applying, and verifying. This means that there are additional components of analogical reasoning developed by Ruppert. Analogical reasoning components in problem-solving depend on the analogical problem is given.
\end{abstract}

\section{INTRODUCTION}

Analogical reasoning is important in learning mathematics (Gentner, 1983; Duit, 1991; Gentner, Holyoak, \& Kokinov, 2001; Holyoak \& Hummel, 2001; Richland, Holyoak, \& Stigler, 2004; Amir-Mofidi, Amiripour, \& Bijan-Zadeh, 2012; Magdas, 2015). Analogical reasoning allows someone to solve problems that are not clear, complex, and new (Melis \& Veloso, 1998; Gentner \& Loewenstein, 2002). When problems that were initially seemingly intricately identified as analogous to a known problem, the problem is more easily resolved. This suggests that analogical reasoning provides benefits in solving difficult and abstract mathematical problems).

Problem-solving using analogical reasoning needs to involve analogical reasoning components. Several studies have discussed the analogical reasoning component in solving a mathematical problem. There are 4 components of analogical reasoning, namely encoding, inferring, mapping, and application (Sternberg, 1977). In addition to these 4 components, Sternberg writes the justification and response components. Sternberg's opinion above by adding some subcomponents in the structuring and verifying components (Ruppert, 2013). Ruppert added that the encoding and inferring components can be called structuring components. Meanwhile, within the justifying and response components are said to be verifying components. Therefore, the components of analogical reasoning according to Ruppert (Ruppert, 2013) are structuring (encoding and inferring), mapping, applying and verifying (justification and response). In addition, Thagard (Thagard, 2005) said that resolving the problem with analogical reasoning through 4 stages, namely: (1) facing the problem (target) to be solved; (2) remember the solution of the problem (source); (3) comparing source and target issues, placing relevant components in correspondence with each other; and (4) adjust to the source problem to generate a solution to the target problem.

While (Eliasmith \& Thagard, 2001) divide stages in analogy reasoning into 3 stages, namely retrieval, mapping, and application. Gentner (Gentner, 1983) also identified stages in analogical reasoning into 3 stages, namely access, mapping, and evaluation and use. In solving the problem, reasoner retrieves or accesses the prior knowledge. Furthermore, the reasoning mapped prior knowledge to the problem at hand. Then, the application applies or evaluates and uses (evaluation and use) the solution to the problem at hand. English (English, 2004) argues that there are 3 stages of analogical reasoning in solving the analogical problem. First, the student must know to generalize the general relational structure of known sources or problems, and, if the source problem must be retrieved from memory. Secondly, students should know to search and be able to identify a relational correspondence between the target problem and the source problem. Third, the student must know what to do with the relational similarity between the source and the target problem. This shows that 
the analogous reasoning stages used by Eliasmith \& Thagard, Gentner, English have similarities in their application.

Analogical reasoning stages adapted by Keane in Donoghue (O’Donoghue, 2004) are the recognition of representation, retrieval, mapping, validation, and induction. These five stages are called the five-stage model of analogy. Retrieval stages are related to memory (memory) and adaptation (adaptation), mapping stages related to reasoning and learning by abstraction, transfer stages associated with creativity and learning by transfer.

The type of analogy used in this study is the analogical problem. The analogical problem is a problem consisting of the source problem and the target problem (English, 2004). The analogical problem used in this study differs from previous researchers (Bernardo, 2001; English, 2004; Assmus, Forster, \& Fritzlar, 2014). Indirect analogical problems have a very different problem between the source and the target problem. Indirect analogical problems used in this study has characteristics such as (1) the mathematical concepts between source problems and target problems different; (2) procedures in solving source problems are used to solve target problems; and (3) the context of source problems and target problems are different. Problem solving between source problems and target problems has similarities, where conceptually and procedurally resolving source problems can be used to solve target problems. Indirect analogical problems have not been used in the study of analogical reasoning (Kristayulita, Nusantara, As'ari, \& Sa'dijah, 2018).

The analogical problem used by Assmus, et al, Bernardo, and English can use analogical reasoning components based on Sternberg and Ruppert. The study developed three instruments related to an indirect analogical problem in which one source problem and two target problem. The problems are an indirect analogical problem. Whether Ruppert component can be applied in solving an indirect analogical problem?. Is there a new component in analogical reasoning in solving an indirect analogical problem?.

\section{RESEARCH METHOD}

This study applied the descriptive qualitative approach. Following are the description of the method used in this study.

\subsection{Participants}

Participants are grade 11 high school students. They have received lessons on quadratic equations. That is, they do have formal training and previous experience with problem-solving quadratic equations. Second, target problems are related to the problem of trigonometric equations and a word problem. Trigonometric equations and word problem used because students are studying them. Overall, participants were 60 students from two schools in Mataran City of Nusa Tenggara Barat, Indonesia.

\subsection{Instruments}

The analogical problem consists of source problems and target problems. First, source problems are related to the problem of quadratic equations. Quadratic equations used because students have completed learning about it; that is, they do have formal training and previous experience with problemsolving quadratic equations. Second, target problems are related to word problems. Word problem used because students are studying them.
Table 1. Source problem and target problem

\begin{tabular}{ll}
\hline \multicolumn{1}{c}{ Types } & \multicolumn{1}{c}{ Quations } \\
\hline $\begin{array}{l}\text { Source } \\
\text { problem }\end{array}$ & Find the roots of the quadratic equation $x^{2}-5 x-6=0$ \\
\hline Target & $\begin{array}{l}\text { 1. Find the value of } x \text { that satisfies } \cos 2 x+6 \sin x+7=0 \\
\text { problem } \\
\text { 2. Volume of a box is } 480 \mathrm{~cm} 3 \text { made from a piece of } \\
\text { cardboard that the form of a rectangle. On each corner of } \\
\text { cardboard will be folded after removing the square-shaped } \\
\text { cardboard section on a } 5 \mathrm{~cm} \text { long side piece of cardboard. } \\
\text { If the width of box is } 4 \mathrm{~cm} \text { longer than its length. How do } \\
\text { you determine the length and width of the base of the box }\end{array}$ \\
\hline
\end{tabular}

\subsection{Procedure and Analysis}

The subject worked on the problem individually. Subjects are asked to carefully read the instructions and answer all questions about the task. Steps of data collected: (1) the subject is working on source problems; furthermore, the results of source problems answers are taken and collected from the students, (2) the subject is working on target problems; and then the results of target problems answers are taken and collected from the students as well, (3) selecting student answer sheets that answer correctly from source problems and target problems, and (4) checking answers to students. Then, data of research result analyze to know the occurrence of analogical reasoning. Based on the results of the analysis and student answers of the analogical problems is described and described process of analogical reasoning that occurred.

\section{RESULTS AND DISCUSSION}

\subsection{Research Results}

There are 35 students who answer source problem are correct (Fig. 1). The first target problem is trigonometri equations, there are 20 students who answer are correct (Figure 2). The second target problem is word problem, there are 30 students who answer are correct (Figure 3).

This study aimed to to provide information analogical reasoning process in solving indirect analogical problems. in addition, to identify the analogical reasoning components in solving indirect analogical problems. To describe component of analogical reasoning on the first analogical problem is represented by subject $\mathrm{S} 1$ and subject $\mathrm{S} 2$. While describing component of analogical reasoning on the second analogical problem is represented by subject S3 and subject S4.

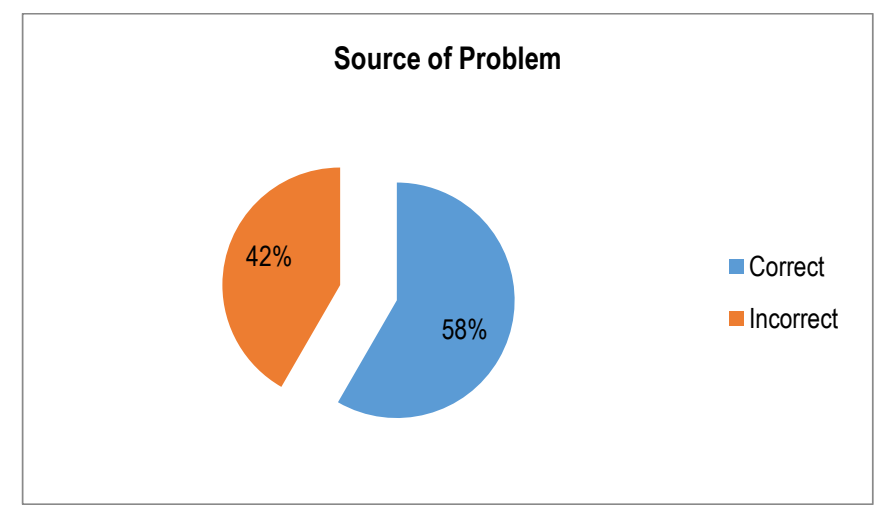

Figure 1. The results of source problems 


\section{First Target Problem}

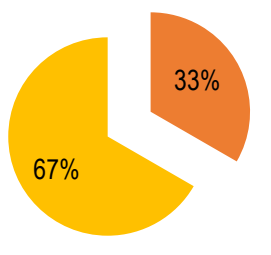

Correct

- Incorrect

Figure 2. The results of the first target problems

\section{Second Target Problem}

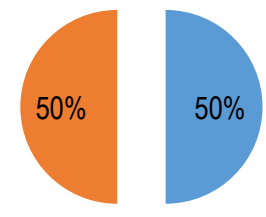

- Correct

- Incorrect

Figure 3. The results of the second target problems

\subsubsection{Analogical Problems: Quadratic Equations and Trigonometric Equation}

The results showed that 20 students did analogical reasoning. The student answers source problems correctly and uses the concept of source problems to solve target problems. Furthermore, some of solving steps used in source problems, students apply to target problems. This can be seen from the result of student's answer on source problems and target problems (Fig. 4 and Fig. 5).

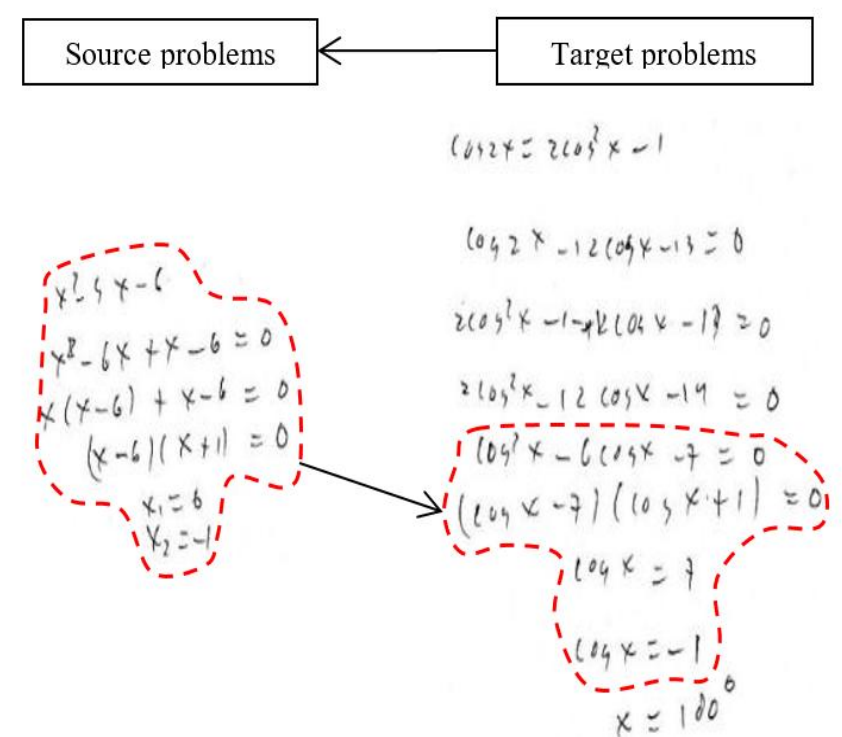

Figure 4. Answers to source problems and target problems for subject $\mathrm{S} 1$

Glossary:

Process of analogical reasoning
Based on Figure 4, subject S1 solves quadratic equations problems (source problems) by using factorization and obtaining correct answers. Furthermore, subject S1 solves trigonometric equations problems(target problems) beginning with changecos $2 x=2 \cos ^{2} x-1$. Subject $\mathrm{S} 1$ substitutes $\cos 2 x=2 \cos ^{2} x-1$ into trigonometric equation so that obtain $\cos ^{2} x-12 \cos x-13=0$. Subject $\mathrm{S} 1$ performs mapping process by making the conclusion that new trigonometric equation obtain is a form of a quadratic equation on trigonometric equations. That is a process of solving target problems using solving process by way solving quadratic equation problems. Structuring process, subject $\mathrm{S} 1$ identifies the problem as it did in resolving source problem. Applying process, subject S1 solves trigonometric quadratic equation problems like solving quadratic equation problems (source problems). And finally, verifying process, subject $\mathrm{S} 1$ finds the value of $\mathrm{x}$ that satisfies for the value $\cos x=-1$ so that subject $\mathrm{S} 1$ obtains the value $x=180$.

Based on Fig. 5, subject S2 solves quadratic equations problems (source problems) by using factorization and obtaining correct answers. Furthermore, subject S2 solves trigonometric equations problems (target problems) beginning with change $\cos 2 x=2 \cos ^{2} x-1$. Subject $\mathrm{S} 1$ substitutes $\cos 2 x=2 \cos ^{2} x-1$ into trigonometric equation so that obtain $2 \cos ^{2} x-12 \cos x-14=0$ then $\cos ^{2} x-6 \cos x-7=0$. Then subject S2 gives $\cos x=A$ obtained $A^{2}-6 A-7=0$. Subject S2 performs mapping process by making the conclusion that new trigonometric equation obtained is a form of a quadratic equation on trigonometric equations. And then, subject S2 obtain new quadratic equation. That is a process of solving target problems using solving process by way solving quadratic equation problems. Structuring process, subject S1 identifies the problem as it did in resolving source problem. Applying process, subject $\mathrm{S} 1$ solves trigonometric quadratic equation problems like solving quadratic equation problems (source problems). And finally, verifying process, subject S2 finds the value of $\mathrm{x}$ that satisfies for the value $\cos x=-1$. Subject $\mathrm{S} 2$ obtains value $x=\pi, 540, \ldots$ Subject S2 obtains the value $x=\pi$.

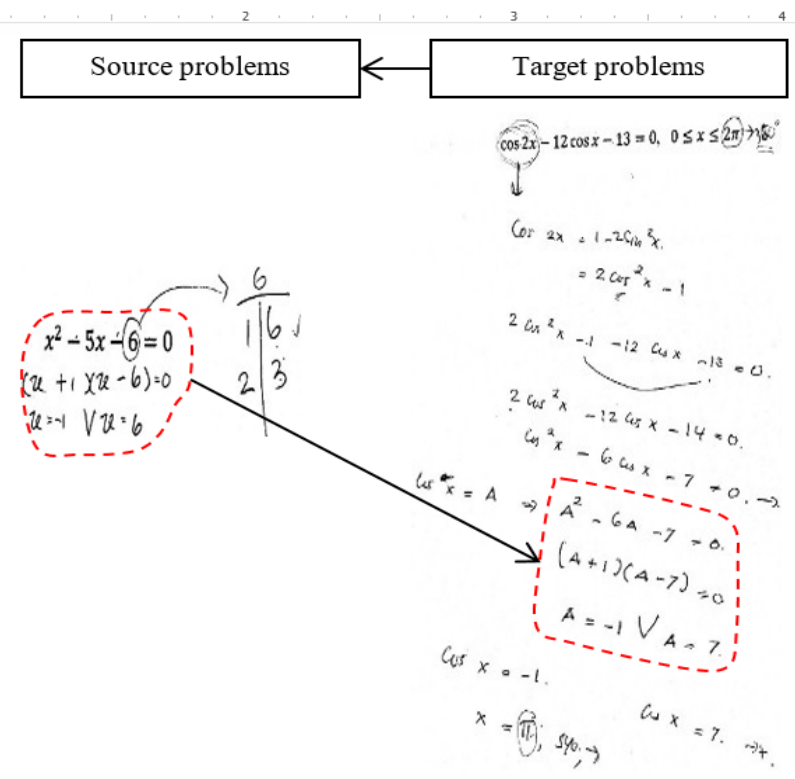

Figure 5. Answers to source problems and target problems for subject $\mathrm{S} 2$

Glossary: 
Based on the work of subject S1 and subject S2, solving analogical problems begins by recognizing the similarity between target problem and source problem. Target problems do not explicitly resemble source problems. Before structuring process, target problems needs to be representation modified to form problems similar to source problems: that is, there is one stage in solving target problems is representation. The aim of changing target problems like source problems. And then, structuring proses, to identify new target problems and to find similarity with source problems. Then mapping from target problems to source problems. Applying process, solving source problems steps are mapped one-to-one to problem-solving step. And finally, verifying process to determine the answer to target problems. An analogical problem-solving process between target problems and source problems using analogical reasoning stages (Figure 6).

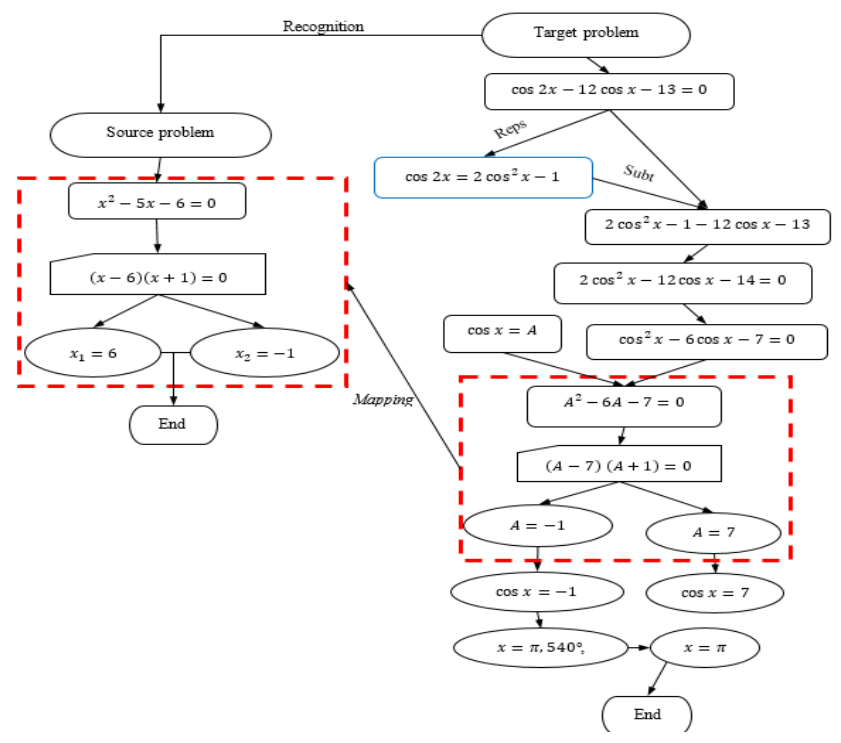

Figure 6. Process of analogical reasoning in analogical reasoning in solving of quadratic equation and trigonometric equation problems.

Description of the coding on the prosess of analogical reasoning in solving of quadratic equation and trigonometric equation problems Fig. 6 (Table 2).

Table 2. Description of the coding on the prosess of analogical reasoning in solving of quadratic equation and trigonometric equation problems

\begin{tabular}{lll}
\hline \multicolumn{2}{c}{ Term } \\
\hline Start/End & \\
\hline Representation process & \\
\hline Structuring process & \\
\hline Mapping process & \\
\hline Applying process & \\
\hline Verifying process & Reps \\
\hline Activity process & \multicolumn{2}{c}{ Subt } \\
\hline Representation & \\
\hline Subtitution & \\
\hline
\end{tabular}

\subsubsection{Analogical Problems: Quadratic Equation and Word Problem}

The results showed that 30 students did analogical reasoning. The student answers source problems correctly and uses the concept of source problems to solve target problems. Furthermore, some of solving steps used in source problems, students apply to target problems. This can be seen from the result of student's answer on source problems and target problems (Figure 7 and Figure 8).

Based on Figure 7, subject S3 solves quadratic equations problems (source problems) by using factorization and obtaining correct answers. Furthermore, the S3 subject solves the problem word problem (target problem). Subject S3 performs a representation beginning to draw a rectangle by making a dashed line of square area at each corner. Subject S3 write box volume $480 \mathrm{~cm}^{3}, l=4+p, t=5$ because lenght removed. Subject S3 writes the volume formula of boxes $p \times l \times t=480 \mathrm{~cm}^{3}$ and $t=5$ because part removed size 5 . Then $p \times l=\frac{480}{5}$. Next $p \times l=96$. Next $p \times(p+4)=96$. Then $p^{2}+4 p=96$. Then we get the equation $p^{2}+4 p-96=0$. Subject S3 performs mapping process by making the conclusion that new word problem obtain is a form of a quadratic equation on word problem. That is a process of solving target problems using solving process by way solving quadratic equation problems. Structuring process, subject S3 identifies the problem as it did in resolving source problem. Applying process, subject S3 solves quadratic equation problems like solving quadratic equation problems (source problems). And finally, verifying process, subject S3 finds lenght of box is 8 and widt of box is 12 .

Based on Figure 8, subject S4 solves quadratic equations problems (source problems) by using factorization and obtaining correct answers. Furthermore, the S4 subject solves the problem word problem (target problem). Subject S4 performs a representation beginning to draw a rectangle by making a dashed line of square area at each corner. Subject $\mathrm{S} 4$ write box volume $480 \mathrm{~cm}^{3}$. Subject S4 writes the volume formula of boxes $p \cdot l \cdot t=480 \mathrm{~cm}^{3}$. Then $x \cdot(x+4) \cdot 5=480 \mathrm{~cm}^{3}$. Next $\left(x^{2}+4 x\right) \cdot 5=480 \mathrm{~cm}^{3}$. Then we get the equation $5 x^{2}+$ $20 x-480=0 \mathrm{~cm}^{3}$. Subject $\mathrm{S} 4$ obtains the equation and divides the equation by 5 obtained $x^{2}+4 x-96=0$. Subject S4 performs mapping process by making the conclusion that new word problem obtain is a form of a quadratic equation on word problem. That is a process of solving target problems using solving process by way solving quadratic equation problems. Structuring process, subject S4 identifies the problem as it did in resolving source problem. Applying process, subject S4 solves quadratic equation problems like solving quadratic equation problems (source problems). And finally, verifying process, subject S3 finds lenght of box is 8 and widt of box is 12. 


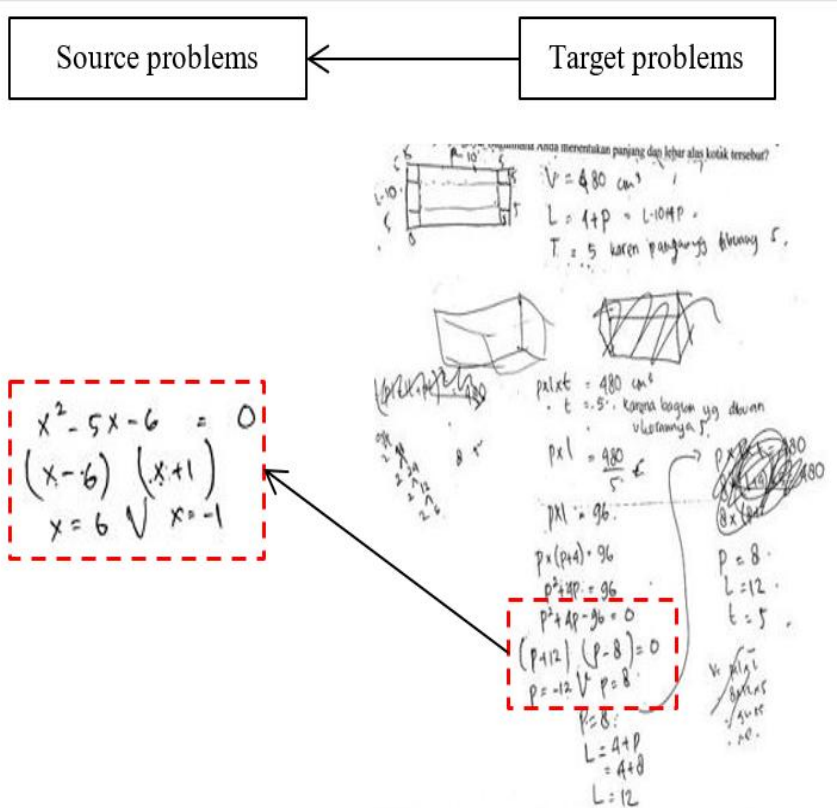

Figure 7. Answers to source problems and target problems for subject S3

Glossary:

$\longrightarrow$ : Process of analogical reasoning

Based on the work of subject S3 and subject S4, solving analogical problems begins by recognizing the similarity between target problem and source problem. Target problems do not explicitly resemble source problems. Before structuring process, target problems needs to be representation modified to form problems similar to source problems: that is, there is one stage in solving target problems is representation. The aim of changing target problems like source problems. And then, structuring process, to identify new target problems and to find similarity with source problems. Then mapping from target problems to source problems. Applying process, solving source problems steps are mapped one-to-one to problemsolving step. And finally, verifying process to determine the answer to target problems. An analogical problem-solving process between target problems and source problems using analogical reasoning stages (Figure 9).

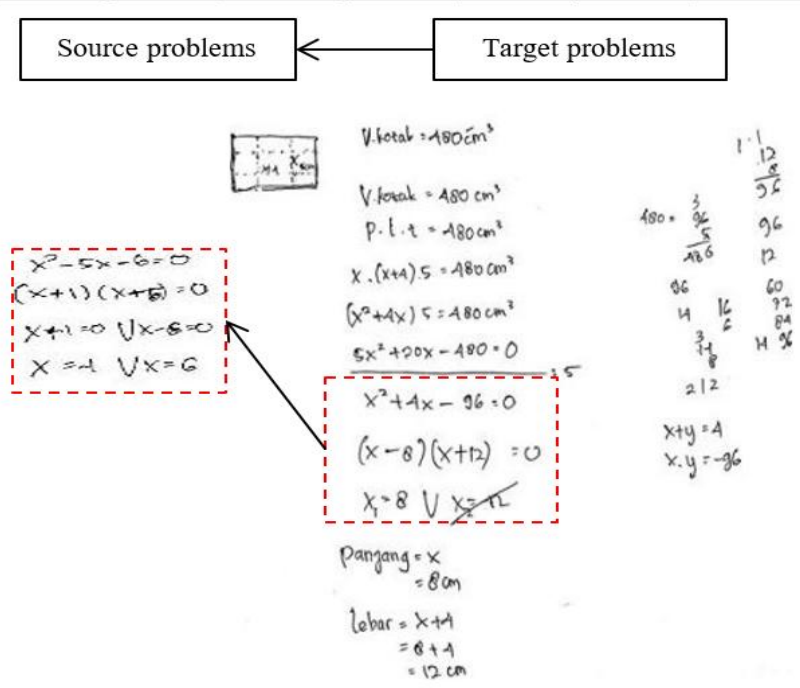

Figure 8. Answers to source problems and target problems for subject S4.
Glossary:

: Process of analogical reasoning

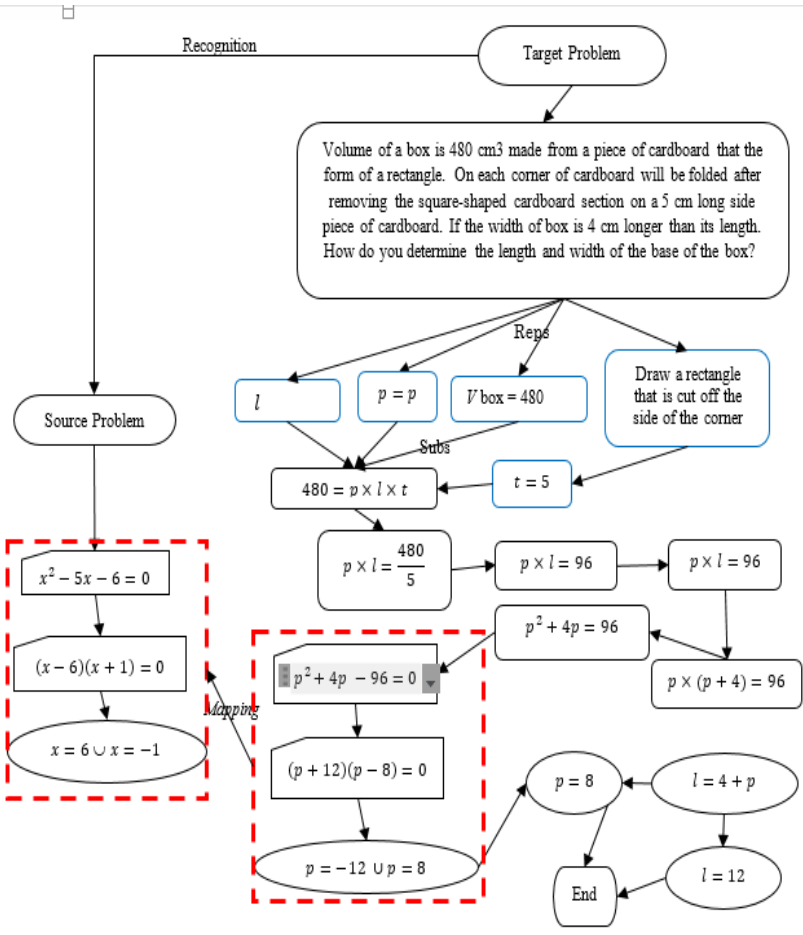

Figure 9. Process of analogical reasoning in analogical reasoning in solving of quadratic equation and word problems

Description of the coding on the prosess of analogical reasoning in solving of quadratic equation and trigonometric equation problems Figure 9 (Table 3).

Table 3. Description coding in the process of analogical reasoning in solving of quadratic equation and word problems

\begin{tabular}{ll}
\hline \multicolumn{1}{c}{ Term } \\
\hline Start/End
\end{tabular}

\subsection{Discussion}

The results show that there are additional stages in solving indirect analogical problems. The additional stages are representation and mathematical modeling. So the stages proposed by (Ruppert, 2013) to be representation and mathematical modeling, structuring, mapping, applying, and verifying. The analogical reasoning stage in resolving indirect analogical problems (Figure 10). 


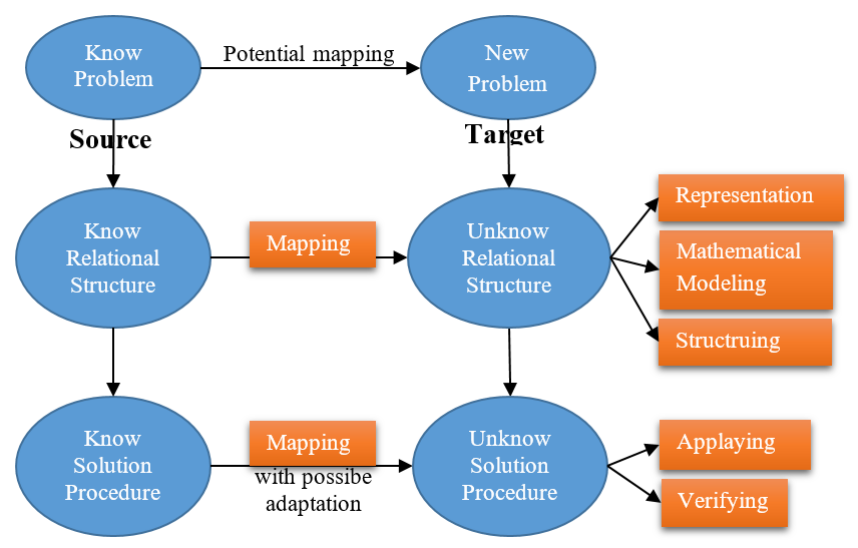

Figure 10. Indirect analogical reasoning

This is different from the stages already expressed by the previous expert (Sternberg, 1977; Ruppert, 2013). According (Sternberg, 1977) there are 4 stages of analogical reasoning that is encoding, inferring, mapping, and aplication. While (Ruppert, 2013) mentions analogical reasoning stages consisting of structuring (encoding and inferring), mapping, applying, and verifying (justification and response). The stages discussed by Ruppert are the development of the Sternberg analogical reasoning stage.

This can be explained by the different types or forms of problems from previous studies. Previous research, source problems and target problems basically have the same type or form (Bernardo, 2001; English, 2004; Assmus et al., 2014). In this research, the source problem is symbolic and the target problem is symbolic and verbal (word problem). Furthermore, the source problem is related to the matter of quadratic equations while the target problem is related to the material of the trigonometric equations and the volume of the building of the beam space. Kristayulita, Nusantara, As'ari, \& Sa'dijah (2018) indirect analogical problems need additional steps in process or analogical reasoning. The additional step is reprsentation.

Representation of a problem is a representation of a text on a problem. According to (Whitten \& Graesser, 2003), text representation is a cognitive representation that has several references to elements, features, or structural patterns in explicit text. In representing the text on the problem of having the levels. According to Graesser, Millis, \& Zwaan and Kintsch in (Whitten \& Graesser, 2003) there are 5 levels in presenting text in the word problem: (1) the surface code retains the exact words and syntax of the text; (2) the textbase consists of words in proportional form, but ignores the exact words and syntax; (3) the situation model (or mental model) is the nonlinguistic referential content present in the text; (4) the communication is defined as capturing the pragmatic context that frames the message in the text; and (5) the related genre assigns the text to one or more rhetorical categories and uses the selected text genre to lead to an understanding

The text representation of the word problem is very important since textual understanding is a fundamental component of problem-solving in an analysis that provides psychomotor judgments on some processing components (Whitten \& Graesser, 2003). The problem-solving process word problem will work if the problem solver does two important things: the understanding of the text that results in the mental representation of the problem and the problem solving where the solver actually calculates the answer. So in the process of solving the problem word problem, the solver needs to have 7 capabilities of text understanding, problem representation, problem categorization, solution estimation, solution planning, procedure evaluation, and self-evaluation.

\section{CONCLUSION}

The results showed that there are stages of representation and mathematical modeling in solving indirect analogical problems. Analogical reasoning stages by Ruppert to be representation and mathematical modeling, structuring, mapping, applying, and verifying. This analogical reasoning stage used in solving indirect analogical problems. Further study is suggested to use mathematical problems that have similarities to the problems in this study. In addition, it is advisable to conduct research related to analogical reasoning error of the student in solving indirect analogical problems..

\section{ACKNOWLEDGEMENTS}

The author is thankful to teachers who helps in providing classes for retrieved data and to students of class XI at two schools (High School 5 Mataram and High School 8 Mataram, 2017) in Mataram city of Nusa Tenggara Barat, Indonesia for participation as respondents in retrieved data. And also to the researchers who shared there their valuable results, without which this study would not have been possible.

\section{REFERENCES}

Amir-Mofidi, S., Amiripour, P., \& Bijan-Zadeh, M. H. (2012). Instruction of mathematical concepts through analogical reasoning skills. Indian Journal of Science and Technology, 5(6), 2916-2922.

Assmus, D., Forster, F., \& Fritzlar, T. (2014). Analogizing during mathematical problem solving-theoretical and empirical considerations. In Proceeding of the Joint Meeting of PME (Vol. 38, pp. 73-80). Retrieved from https://iris.unito.it/retrieve/handle/2318/1620514/28 5455/PME38-2014\%20Vancouver\%202.pdf\#page $=83$

Bernardo, A. B. I. (2001). Analogical Problem Construction and Transfer in Mathematical Problem Solving. Educational Psychology, 21(2), 137-150. https://doi.org/10.1080/01443410020043841

Duit, R. (1991). On the role of analogies and metaphors in learning science. Science Education, 75(6), 649-672.

Eliasmith, C., \& Thagard, P. (2001). Integrating structure and meaning: A distributed model of analogical mapping. Cognitive Science, 25(2), 245-286.

English, L. D. (2004). Mathematical and analogical reasoning of young learners. Routledge. Retrieved from https: / / www.google.com/books?hl=en\&lr=\&id=GCqRAg AAQBAJ\&oi=fnd\&pg=PP1\&dq=Mathematical + and + analo gical+reasoning+of+young+learners\&ots=oXoAt3Ad_U\&si g=DVbtiQwggdS6COnnz916j6iqZF4

Gentner, D. (1983). Structure-mapping: A theoretical framework for analogy. Cognitive Science, 7(2), 155-170.

Gentner, D., Holyoak, K. J., \& Kokinov, B. N. (Eds.). (2001). The analogical mind: perspectives from cognitive science. Cambridge, Mass: MIT Press.

Gentner, D., \& Loewenstein, J. (2002). Relational language and relational thought. Language, Literacy, and 
Cognitive Development: The Development and Consequences of Symbolic Communication, 87-120.

Holyoak, K. J., \& Hummel, J. E. (2001). Toward an understanding of analogy within a biological symbol system. The Analogical Mind: Perspectives from Cognitive Science, 161-195.

Kristayulita, K., Nusantara, T., As'ari, A. R., \& Sa'dijah, C. (2018). Identification of Students Errors in Solving Indirect Analogical Problems Based on Analogical Reasoning Components. In Journal of Physics: Conference Series (Vol. 1028, p. 012154). IOP Publishing.

Magdas, I. (2015). Analogical Reasoning in Geometry Education. Acta Didactica Napocensia, 8(1), 57-65.

Melis, E., \& Veloso, M. (1998). Analogy in problem solving. In Handbook of practical reasoning: Computational and theoretical aspects. Citeseer. Retrieved from http://citeseerx.ist.psu.edu/viewdoc/summary?doi=10. 1.1.51.8553

O’Donoghue, J. (2004). Finding Novel Analogies. University College Dublin. Retrieved from: http://www.cs.nuim.ie/ dod/pubs/05-thesis.pdf

Richland, L. E., Holyoak, K. J., \& Stigler, J. W. (2004). Analogy use in eighth-grade mathematics classrooms. Cognition and Instruction, 22(1), 37-60.

Ruppert, M. (2013). Ways of analogical reasoning-thought processes in an example based learning environment. Erscheint in: Proceedings of the CERME, 8. Retrieved from http://cerme8.metu.edu.tr/wgpapers/WG1/WG1_Rupp ert.pdf

Sternberg, R. J. (1977). Component processes in analogical reasoning. Psychological Review, 84(4), 353.

Thagard, P. (2005). Mind: introduction to cognitive science (2nd ed). Cambridge, Mass: MIT Press.

Whitten, S., \& Graesser, A. C. (2003). Comprehension of text in problem solving. The Psychology of Problem Solving, 207-229. 